\title{
Direct printing of polymer microstructures on flat and spherical surfaces using a letterpress technique
}

\author{
Scott M. Miller and Sandra M. Troian ${ }^{\text {a) }}$ \\ Microfluidic Research and Engineering Laboratory, Department of Chemical Engineering, \\ Princeton University, Princeton, New Jersey 08544 \\ Sigurd Wagner \\ Department of Electrical Engineering and Center for Photonics and Optoelectronic Materials, \\ Princeton University, Princeton, New Jersey 08544
}

(Received 1 April 2002; accepted 16 September 2002)

\begin{abstract}
We have developed a letterpress technique capable of printing polymer films with micrometer scale feature sizes onto flat or spherically shaped nonporous substrates. This printing technique deposits polymer only in desired regions thereby eliminating subsequent developing and subtraction steps. Flat or curved printing plates, which are fabricated from either rigid or deformable materials, are used to transfer thin molten polymer films onto flat target substrates. By deforming the printing plates into a spherical shape, it is also possible to print patterned films onto the concave side of a spherically deformed target substrate. These printed films serve as good resists for both wet chemical etching and reactive ion etching. Interferometric measurements of the polymer film thickness are used to probe physical mechanisms affecting printing instabilities, pattern fidelity, and edge resolution. Our experimental study indicates that this letterpress technique may prove suitable for high-throughput device fabrication involving large-area microelectronics. (c) 2002 American Vacuum Society. [DOI: 10.1116/1.1520554]
\end{abstract}

\section{INTRODUCTION}

Contact printing techniques, which combine the advantages of good resolution, rapid speed, and low cost, are typically used to pattern high-volume graphic and typographic products. Printing methods such as embossing, gravure, letterpress, offset, and flexography can produce patterns with feature resolution as small as $\sim 40 \mu \mathrm{m}$ onto areas as large as square meters. The printing is extremely rapid and can achieve coverage rates on the order of $30 \mathrm{~m}^{2} / \mathrm{s}^{1}$

Fabrication of microelectronic devices and microelectromechanical systems, which require much higher resolution, relies instead on photolithography. Patterns as small as 100 $\mathrm{nm}$ are created in a thin photosensitive polymer film that is exposed to ultraviolet light through a partially opaque mask and then developed to remove unwanted polymer. The pattern defined in the polymer film is then transferred to the underlying material layers by etching. Large areas are patterned by step-and-repeat procedures, a time consuming and expensive subtractive process. In addition, photolithography lends itself primarily to flat surfaces. Cylindrically or spherical curved substrates pose a significant challenge.

While no other commercial technique today can compete with the ultrahigh resolution and pattern definition obtained with photolithography, many emerging applications do not require such small feature sizes. Microelectronic assemblies fabricated in large-area format (on the order of square meters or larger), like light emitting displays or embedded sensor and actuator arrays, are under development in many industrial and academic laboratories. The capability to fabricate

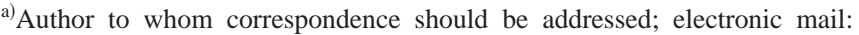
stroian@princeton.edu
}

such displays and arrays directly on flat, cylindrical, spherical, and flexible substrates would be very attractive. Contact printing offers this potential, with the additional benefits of rapid throughput and low cost.

Research groups have investigated a variety of patterning techniques. Kumar et al. ${ }^{2}$ have shown that alkane thiol monolayers can be patterned on gold by microcontact printing. In a two-step process, the patterned thiol monolayer is used as an etch mask for the gold film, which in turn can serve as an etch mask for the underlying device layer to be patterned. Tiberio et al. ${ }^{3}$ have also used alkane thiol monolayers patterned by electron-beam lithography, as etch masks for gallium arsenide. Mikami et al. ${ }^{4}$ introduced a gravureoffset technique to print ink that serves as a photo-mask on a photoresist film used for fabrication of thin-film transistors (TFTs). Lahti et al. ${ }^{5}$ have employed a similar technique to directly print conductive thick-film inks. Garnier ${ }^{6}$ and Rogers $e t$ al. $^{7}$ have each patterned elements of TFTs using screen printing. Gleskova and Wagner ${ }^{8}$ have studied laser printing as a method for patterning resist layers for TFT fabrication and have recently used this method for depositing deposit thick-film $(10-25 \mu \mathrm{m})$ polymer dielectrics. ${ }^{9}$ Hebner and co-workers ${ }^{10}$ have fabricated polymer patterns for three color organic light emitting diodes by inkjet printing. Sirringhaus et al. ${ }^{11}$ have also used inkjet printing over prepatterned polyimide films to fabricate elements of organic TFTs. Hong and Wagner ${ }^{12}$ inkjet printed copper organic precursors for source/ drain metallization of amorphous silicon TFTs. Other techniques, which do not involve conventional printing methods, have been devised for pattern transfer, including the micromolding in capillaries (MIMIC) process introduced by Xia et al. ${ }^{13}$ and Rogers et al. ${ }^{7}$ 
There is far less published work on high-resolution printing onto curved surfaces. Erhardt et al. ${ }^{14}$ have patterned TFTs on gently curved surfaces using the MIMIC method. Jackman et al. ${ }^{15}$ and Rogers et al. ${ }^{16}$ have used microcontact printing to pattern thin metal films onto the cylindrical surface of an optical fiber. Besides the focus on methods of pattern transfer, several groups are developing specialized functional materials, like polymer semiconductors, to enhance compatibility with the newer, nonphotolithographic methods of device fabrication. ${ }^{17}$

This article describes the development of a letterpress technique capable of printing polymer films with micrometer scale feature sizes onto flat or spherically shaped nonporous substrates. While conventional photolithographic and etching techniques are used to produce the original printing plates, these stamps are then repeatedly used to print thin polymer melt films onto rigid or flexible substrates. This additive printing technique eliminates the usual post-printing developing steps for material removal. The printed films serve as good resists for both wet chemical etching and reactive ion etching. Ellipsometric and interferometric measurements of the thickness of the printed film are used to probe physical mechanisms affecting printing instabilities, pattern fidelity, and edge resolution.

\section{LETTERPRESS PRINTING TECHNIQUE}

The letterpress printing method consists of two principal steps, as illustrated in Fig. 1. A printing plate or stamp with features defined in relief (i.e., the designed pattern projects above the surrounding areas) is first inked by pressing the stamp against a carrier substrate coated with a thin molten polymer film. The stamp is then contacted to a target substrate and pressure is applied to transfer the polymer film. During the inking and printing steps, the polymer film is heated above its glass transition temperature $T_{g}$ (or melting temperature $T_{m}$ for crystalline polymers) to decrease the viscosity and allow facile flow and transfer. The heat source is removed following separation of the printing plate and target substrate to fix the pattern by allowing the polymer to cool, as illustrated in Fig. 1(b).

This printing method is less sensitive to the surface chemistry of the target substrate than monolayer stamping by microcontact printing, ${ }^{2}$ which requires the formation of chemical bonds between the monolayer and substrate. Polymer printing can also be used to form relatively thick and robust etch resists which protect underlying layers better than a monolayer coating. A variety of materials can therefore be printed onto heterogeneous substrates, a common requirement in microelectronic fabrication.

\section{EXPERIMENTAL METHODS}

\section{A. Materials selection and preparation}

Pattern transfer is more easily achieved if the ink remains liquid-like during the printing process. If the material is later to serve as an etch resist, however, it must become solid at the conditions used for further processing. One approach is (a)

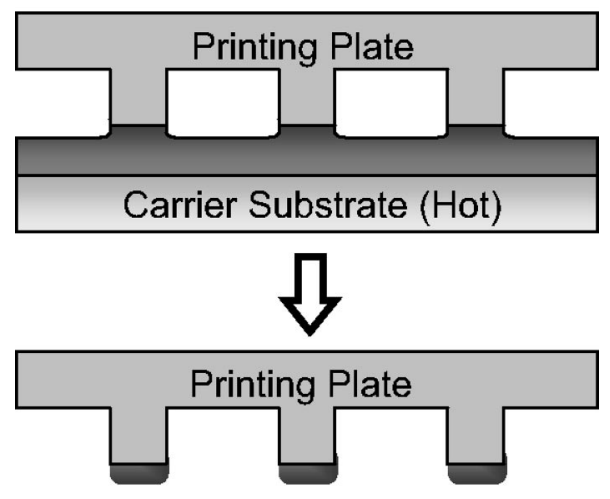

(b)

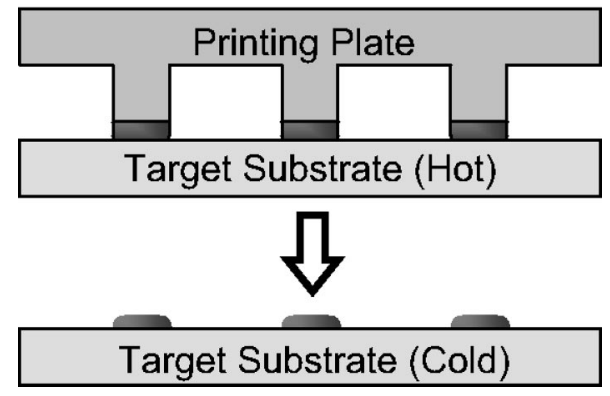

FIG. 1. Schematic illustration of the letterpress printing method. (a) Polymer ink is deposited onto the raised features of the printing plate by contacting the plate to a thin polymer melt film supported on a carrier substrate. The initial film is spin coated from solution onto the carrier substrate. (b) The polymer image is directly transferred from the printing plate to a target substrate in additive fashion.

to use a prepolymer ink that can be cured during or immediately after printing. ${ }^{18} \mathrm{~A}$ second approach is to use a thermoplastic polymer, which can be heated to a temperature above $T_{g}$ during inking and transfer but then rapidly cooled to fix the pattern and prevent undesirable spreading.

The primary ink used in these studies was polystyrene (PS), which has several desirable properties. Since the glass transition temperature $T_{g} \approx 95^{\circ} \mathrm{C}$, it can easily be heated to melt the polymer and then cooled to the solid state for further processing. Polystyrene is also resistant to many of the wet etchants used in device fabrication, and its high carbon fraction also makes it robust to reactive ion etching. ${ }^{19,20}$

To ensure that PS remained liquid-like during inking and transfer, all printing steps were performed at a temperature $T_{\text {process }}=180^{\circ} \mathrm{C} \gg T_{g}$. The polymer molecular weight, $M_{w}$ $=0.8-5 \mathrm{~kg} / \mathrm{mol}$, was chosen to lie well below the entanglement molecular weight $M_{e}=18 \mathrm{~kg} / \mathrm{mol}$ to reduce the viscosity. In this range of molecular weight at $T_{\text {process }}$, PS has a viscosity $^{21} \quad \eta \approx 210 \mathrm{mPas}$ and a surface tension ${ }^{22} \gamma$ $=29.0 \mathrm{mN} / \mathrm{m}$. Since PS readily dissolves in toluene and other solvents, it can be spin coated onto a carrier substrate and easily removed from the target after use as an etch mask. As part of our study, we have successfully printed other polymers, including polyethylene and poly(ethylene oxide) 
by using appropriate solvents and adjusting the process temperatures accordingly.

All metal films were deposited using an electron-beam evaporator (Innovative Systems) at $10^{-6}$ Torr. The gold films were etched with TFA Gold Etchant (Transene Co., Inc). Chromium films were etched with CR-7 photomask etchant (Cyantek Corp.). Silicon nitride films were deposited by plasma-enhanced chemical vapor deposition (PECVD) in a Plasmatherm 790 system at 900 mTorr and $250{ }^{\circ} \mathrm{C}$ with a power setting of $0.08 \mathrm{~W} / \mathrm{cm}^{2}$ and gas flow rates of $\mathrm{N}_{2}, \mathrm{SiH}_{4}$, and $\mathrm{NH}_{3}$ of 150,110 , and $2 \mathrm{sccm}$, respectively. The silicon nitride was etched with a Plasmatherm 790 reactive ion etching (RIE) system using $35 \mathrm{sccm} \mathrm{CF}_{4}$ and $5 \mathrm{sccm} \mathrm{O}_{2}$ at 100 $\mathrm{mTorr}$ and $0.2 \mathrm{~W} / \mathrm{cm}^{2}$. The polyimide foils (50 $\mu \mathrm{m}$ Kapton ${ }^{\circledR}$ E from DuPont) were oxygen plasma etched at $100 \mathrm{mTorr}$ using $40 \mathrm{sccm} \mathrm{O}_{2}$ and $1.0 \mathrm{~W} / \mathrm{cm}^{2}$ with silicon nitride etch masks. Silicon wafers were wet etched with a $20 \mathrm{wt} \% \mathrm{KOH}$ aqueous solution at $75^{\circ} \mathrm{C}$ to produce angled sidewalls. Vertical sidewalls were obtained by etching in a Plasmatherm SLR series RIE at $60 \mathrm{sccm} \mathrm{SF} 6$ and $40 \mathrm{sccm} \mathrm{CCl}_{2} \mathrm{~F}_{2}$ at 30 mTorr and $0.037 \mathrm{~W} / \mathrm{cm}^{2}$. Glass slides were etched with hydrofluoric acid.

\section{B. Stamp fabrication}

Both rigid and flexible stamps were used as printing plates with relief depths of 5-12 $\mu \mathrm{m}$. The rigid stamps were fabricated from [100] silicon wafers or glass slides; the flexible ones were made from $50-\mu \mathrm{m}$-thick polyimide foils. These stamps were prepared by standard photolithographic procedures and etched according to the recipes above. We observed no significant degradation of the stamps or printed patterns with multiple use, as inspected with an optical microscope.

\section{Film thickness measurements}

The relief depth of the patterned stamps was measured with a Veeco Dektak 3 stylus profilometer. The thickness of spin-coated films was obtained with a Gaertner Scientific L116C Ellipsometer in two angle or two wavelength mode; the index of refraction of the polystyrene films was measured to be 1.59 , in agreement with published values. ${ }^{23}$ Printed and solidified PS patterns exhibited nonuniformities in film thickness, as described in Sec. V. The degree of nonuniformity depended on the printed feature size. The thickness of these structured films was determined from optical interference images obtained with an Olympus BX60 microscope equipped with a $550 \mathrm{~nm}$ green optical bandpass filter. Constructive and destructive interference conditions led to dark fringes at thicknesses

$$
h_{i}=\frac{\lambda}{4 n}(2 i-1) \quad \text { for } i=1,2,3, \ldots,
$$

and bright fringes at thicknesses

$$
h_{i}=\frac{\lambda i}{2 n} \text { for } i=1,2,3, \ldots,
$$

where $\lambda=550 \mathrm{~nm}$ is the optical wavelength, $n$ is the index of refraction of the polymer film, and $i$ is the fringe number. The first dark fringe therefore represents a film thickness $h$ $=86 \mathrm{~nm}$. The average film thickness of a patterned polymer film was estimated from optical micrographs according to the following relation:

$$
h_{\text {avg }}=\frac{\sum h_{i} \times \text { Area }_{i}}{\sum \text { Area }_{i}},
$$

where $\mathrm{Area}_{i}$ denotes the total area enclosed by either a bright or dark fringe. Image analysis using "blob analysis" algorithms provided by the Epix XCap software was used to identify the fringe contours.

\section{PRINTING PROCEDURES}

\section{A. Printing onto flat surfaces}

The polymer film deposited onto the carrier substrate (Fig. 1) was formed by spin coating a solution containing PS and toluene. Alternative coating techniques, such as dip coating or roller coating, are particularly suitable for deposition on larger area substrates. For the process parameters used in this study, the spin coating method yielded a polymer thickness of approximately $0.5-1 \mu \mathrm{m}$. The coated carrier substrate was heated to $180^{\circ} \mathrm{C}$ and then the printing plate was pressed against the polymer film. Upon separation, the raised features of the stamp were left coated with a polymer film approximately 250-500 nm thick. As discussed in Sec. V A, the thickness of this residual film is found to be independent of the feature size.

The coated stamp was then contacted to a target substrate, which was heated to the same process temperature. This produces a patterned film of approximately 100-300 nm thickness on the target substrate. Sample to sample variations in stamp and target film thickness were mostly caused by the fact that all steps were performed manually. For example, the applied pressure was not always constant nor was it necessarily uniformly distributed. The thickness of the printed patterned films was measured to be approximately half the original thickness of ink that coated the stamp. This result is in agreement with previous studies which have shown that for sufficiently rapid separation of two rigid plates containing an interstitial liquid, the fluid volume partitions equally between the two surfaces irrespective of the equilibrium wetting angles on the plates. ${ }^{24,25}$ Inertial forces dominate over capillary forces in this high speed regime.

The printing plate can either be used as a flat plate or a cylindrical roller. We typically implement a hybrid process in which the flexible printing plate contacts the target substrate in a planar configuration but is then separated at one edge and peeled away during detachment, as would occur with a roller configuration.

\section{B. Printing onto spherical surfaces}

For printing onto spherical surfaces, we inflated thin foils into spherical cap shapes as shown in Fig. 2(a) ${ }^{26}$ The foil was clamped along a circular boundary and a uniform gas 


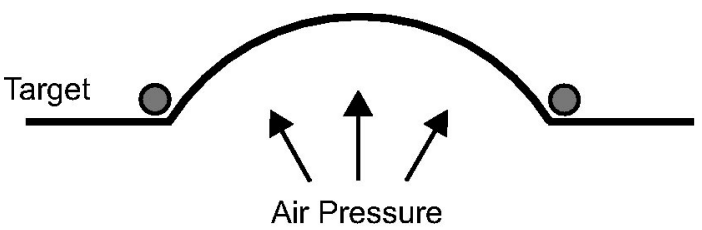

(a)

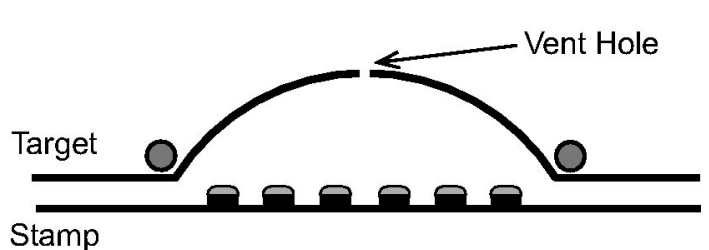

(b)

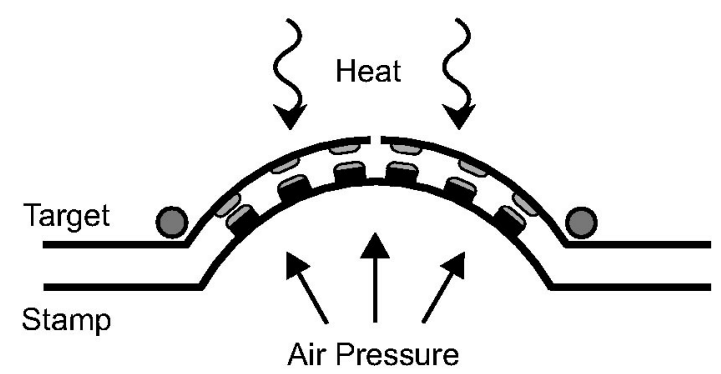

(c)

FIG. 2. Procedure for printing polymer images onto a spherical substrate. (a) The flexible target substrate is first deformed into a spherical cap shape by clamping the foil to an annular ring and inflating the interior with air pressure. (b) An inked printing plate is placed beneath a spherically deformed substrate whose center is perforated to create a vent hole. (c) Gas pressure is applied to inflate the inked printing plate. The plate is made to contact a target substrate, which is heated from above in order to melt the polymer film and allow pattern transfer. Once the pressure is released, the stamp detaches from the target, which remains spherically deformed.

pressure $\left(N_{2}\right)$ was applied to the foil underside. The apparatus allowed control of the applied pressure as well as heating from above using a heat gun. A gas pressure of $50 \mathrm{psi}$ applied to a $50-\mu \mathrm{m}$-thick polyimide foil clamped at a circular diameter of $5 \mathrm{~cm}$ was used to deform the foil into a spherical cap with an opening angle of $66^{\circ}$ (one steradian solid angle). ${ }^{26,27}$ This deformation produced an average radial strain of 5.6\% in the foil.

Printing onto a spherical substrate required slight modification of the procedure used for flat printing. The target substrate was first plastically deformed into a spherical cap shape as shown in Fig. 2(a). We perforated this foil by passing a needle through its apex to create a small vent hole from which trapped air could escape during the printing process [Fig. 2(b)]. A flat and flexible polyimide stamp, which has been inked using the procedure for flat printing, was then placed beneath the spherically deformed target and clamped into the inflation apparatus. The target substrate was heated from above until the temperature of the upper surface reached the process temperature, at which time the stamp was inflated until it made contact everywhere with the target surface [Fig. 2(c)]. As the pressure was removed and the deformation relaxed, the stamp spontaneously separated from the target. Although some residual deformation remained in the stamp after printing, it could be re-used by inking the raised features once again by inflating the stamp into contact with a polymer melt film on a spherical carrier foil, following the procedure just outlined.

\section{RESULTS AND DISCUSSION}

\section{A. Flat target substrates}

Figure 3 shows optical micrographs of a flexible stamp during the three stages of printing onto flat surfaces. Figure 3(a) depicts the original stamp surface made from a polyimide foil, which was patterned by RIE to a depth of $5 \mu \mathrm{m}$ into an array of $75 \mu \mathrm{m}$ squares at a pitch of $120 \mu \mathrm{m}$. The raised features, which are coated with a $200 \mathrm{~nm}$ of PECVD silicon nitride, appear bright against the surrounding recessed regions, which appear dark due the roughening of the etched surface.

Figure 3(b) shows the same stamp immediately after the polymer ink was applied. The variations in gray scale within the square regions are optical interference fringes, indicating that the polymer film thickness is not uniform. The polymer solidifies before surface tension can equilibrate the film profile to a smooth and rounded shape. The interference fringes show several thick lobes of material surrounded by thinner regions. Comparison of this morphology with that obtained when separating two plates containing an interstitial viscous liquid $^{28}$ suggests that the nonuniformities are remnants of a printing instability caused by "viscous fingering." This instability occurs when a less viscous medium like air suddenly and rapidly penetrates a more viscous medium like a polymer melt. ${ }^{29}$ For Newtonian liquids, the wavelength $\Lambda$ of this instability has been estimated to correlate with the various material and process parameters according to $^{28}$

$$
\Lambda=\pi \sqrt{\frac{\gamma h_{\mathrm{avg}}^{2}}{\eta V},}
$$

where $\gamma$ is the polymer melt surface tension, $h_{\text {avg }}$ is the average film thickness, $\eta$ is the polymer melt viscosity, and $V$ the plate separation speed. Using the material constants for PS, a separation speed of $V=1 \mathrm{~cm} / \mathrm{s}$ and film thickness $h_{\text {avg }}=1 \mu \mathrm{m}$ leads to a wavelength estimate of $12 \mu \mathrm{m}$, consistent with the remnant fibril spacing observed in Fig. 3(b).

The nonuniformities in film thickness caused by this dynamic effect are not detrimental to the printing process, but they can nonetheless be annealed away by heating the polymer film to temperatures above $T_{g}$. Figure 3(c) shows the surface of the same sample as in Fig. 3(b) after annealing for $10 \mathrm{~min}$ at $T=180^{\circ} \mathrm{C}$. The interference fringes indicate a gently rounded shape in which the apex height ranges from $865-1125 \mathrm{~nm}$ and the average film thickness from 425-575 $\mathrm{nm}$. As the feature size is decreased below $\Lambda$, the nonuniformities disappear. This is likely due to two effects: Suppression of the instability as well as more rapid surface equilibration by capillary forces for smaller sized features. Figure 4 is a scanning electron micrograph of a PS coated stamp that has smaller and more deeply etched features. The poly- 

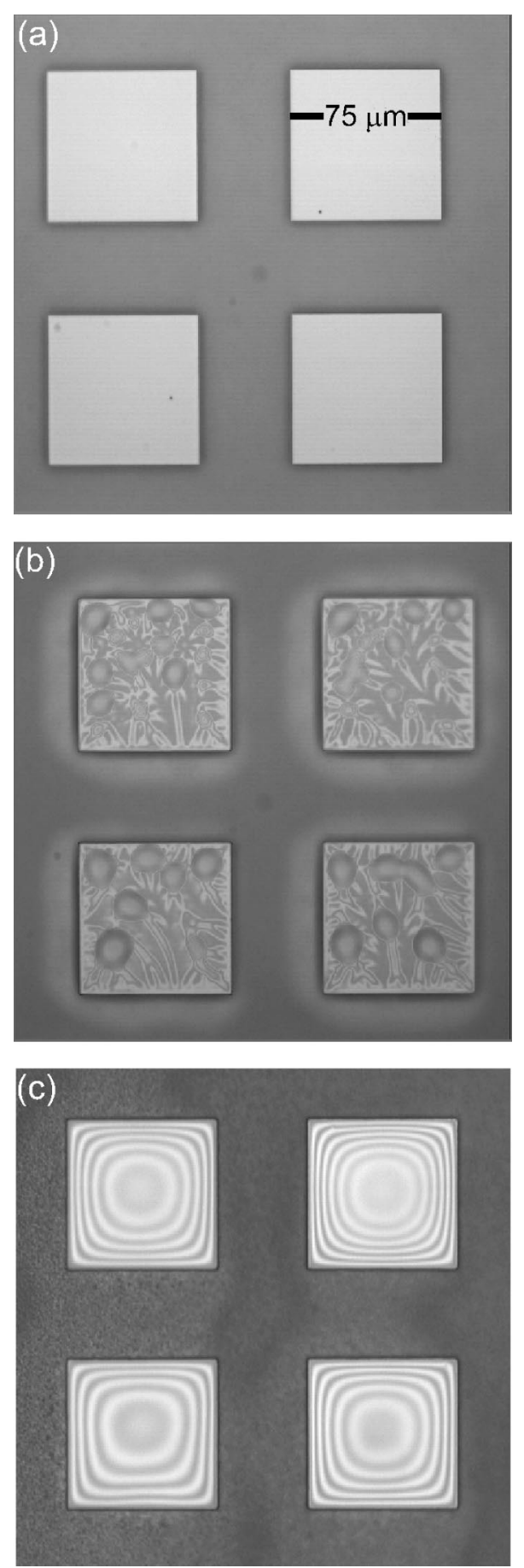

FIG. 3. Optical micrographs of the surface of a polyimide stamp before and after deposition of polystyrene ink. (a) The native stamp surface has been patterned by RIE to a depth of $5 \mu \mathrm{m}$. The raised features, coated with 200 $\mathrm{nm}$ of silicon nitride, appear bright. (b) The same stamp immediately after the polymer ink was applied. Variations in gray scale within the square regions are optical interference fringes that indicate variations in the polymer film thickness. The interior of the square contains numerous polymer mounds surrounded by much thinner regions. (c) The same stamp as in (b) after annealing for $10 \mathrm{~min}$ at $T=180^{\circ} \mathrm{C}$.

mer film that coats the top of each pillar is very thin and smooth. Even with SEM, it is difficult to ascertain whether any polymer spreads to wet the sidewalls of the raised polyimide features.

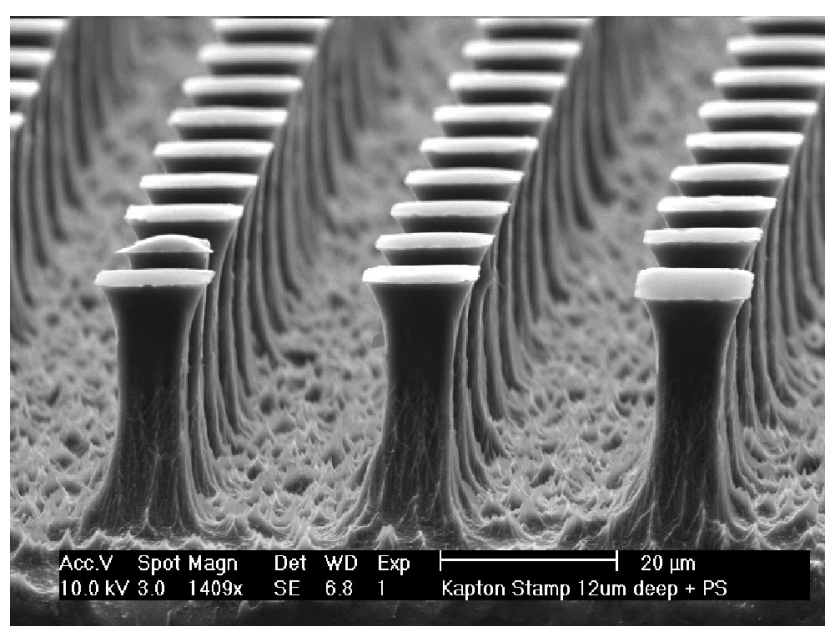

FIG. 4. Cross-sectional scanning electron micrograph of a polyimide printing plate after deposition and annealing of the polystyrene ink. Undercutting is observed with the deep etch used in the fabrication.

Figure 5 shows two different sized squares of PS (40 and $16 \mu \mathrm{m})$ that were printed from a polyimide stamp onto a silicon wafer coated with $200 \mathrm{~nm}$ of silicon nitride. The images to the left, Figs. 5(a) and 5(c), show the surface of the target substrate immediately after printing. The larger printed structures underwent the same viscous instability described earlier upon detachment of the printing plate from the target substrate. The smaller squares do not show these corrugations but instead exhibit smoother rounded profiles. Analysis of the interference fringes for (a) and (c) reveal that the average polymer thickness is independent of the feature size, in

(a)

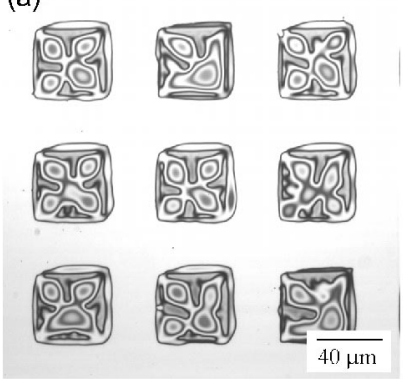

(c)

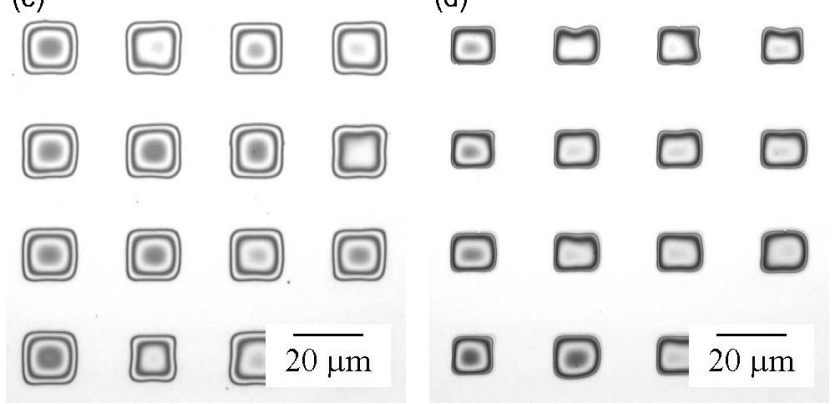

(b)

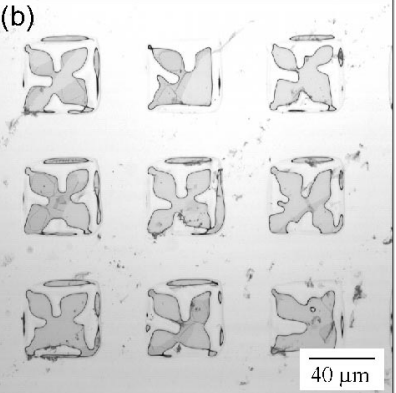

(d)

FIG. 5. Optical micrographs showing the surface of two sizes of polystyrene squares, 40 and $16 \mu \mathrm{m}$, printed from a polyimide stamp onto a silicon wafer coated with $200 \mathrm{~nm}$ silicon nitride. (a) and (c) Target substrate surface immediately after printing. (b) and (d) Same substrates after reactive ion etching of the silicon nitride film. 


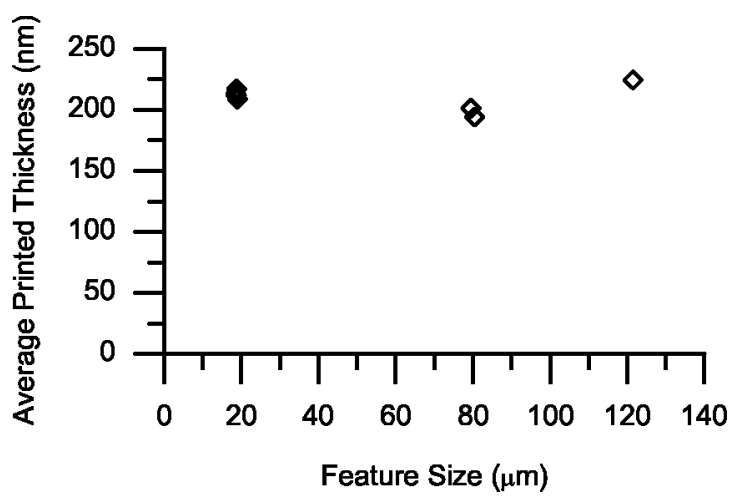

FIG. 6. Interferometric measurements of the average printed film thickness $h_{\text {avg }}$ of a polystyrene square as a function of feature size. The data represents four separate measurements for the smallest feature size and two separate measurements for the larger sizes.

contrast, for example, to printed structures obtained by offset printing. ${ }^{30}$ Results from additional experiments indicate that the thickness of letterpress printed films is rather insensitive to the feature size, as illustrated by the plot in Fig. 6 .

The images to the right in Figs. 5(b) and 5(d), depict the same samples after reactive ion etching of the silicon nitride film. Because the polystyrene mask is slowly etched by this procedure, the thinnest regions of the mask are removed, leading to undesirable etching of the underlying silicon nitride layer. In this way, the nonuniformities in the printed mask are transferred to the silicon nitride. Since the mask with the smaller features shown in Fig. 5(c) did not contain such layer nonuniformities, the etched silicon nitride patterns shown in Fig. 5(d) were reproduced much more faithfully. These smaller feature masks contain dome-shaped PS structures whose thickness is smallest at the edges. This results in the silicon nitride being etched near the feature edges, resulting in squares of silicon nitride that are smaller than the original polymer footprint. This problem can be minimized, however, by improving the selectivity of the etch process through modification of the etch recipe and by leveling the polymer surface before etching. We are currently examining the competition between polymer redistribution within the interior of the printed squares by annealing at $T>T_{g}$ (for purposes of surface leveling), and the spreading of the polymer footprint induced by this process. The polymer profile on even the smallest printed structures, however, is expected to remain somewhat rounded due to surface tension.

The micrographs in Fig. 7 clearly demonstrate the effect of feature size on the film morphology induced by the viscous instability, as the feature size decreases from 120 to $16 \mu \mathrm{m}$. The larger features show additional structure near the edges. As the feature size decreases toward $\Lambda$, the distribution of the mounds is increasingly influenced by these edges. As noted earlier, the corrugations vanish for the smallest feature size shown.

The printed polystyrene films can also be used as masks for wet etching. Figure 8 shows a patterned array of 2.5 - $\mu \mathrm{m}$-diam gold dots with a pitch of $14 \mu \mathrm{m}$ formed on a glass slide by printing and wet etching. The gold, which sits
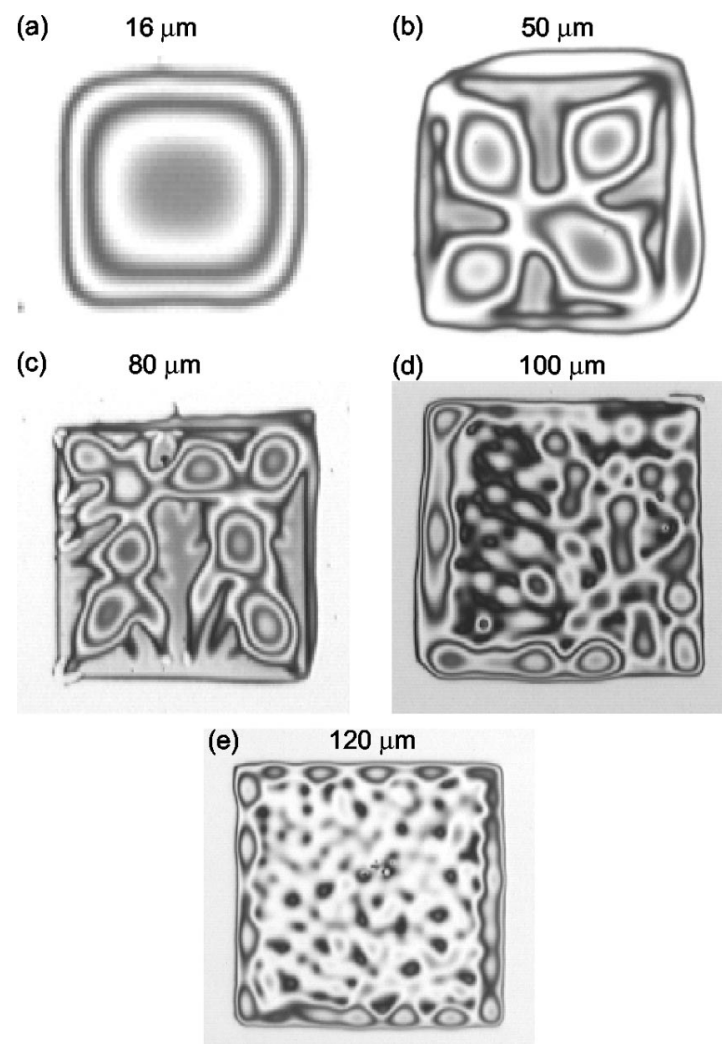

FIG. 7. Optical micrographs of the surface of printed polystyrene squares ranging in size from $16-120 \mu \mathrm{m}$. The viscous fingering instability described in the text is suppressed and the surface considerably smooths as the feature size approaches the estimated instability wavelength.

atop of a $10 \mathrm{~nm}$ chromium adhesion layer, is $90 \mathrm{~nm}$ in thickness. Unlike the structures formed by reactive ion etching, there was no reduction in feature size upon wet etching since PS is much more resistant to attack by the wet etching solution than by RIE using $\mathrm{CF}_{4} / \mathrm{O}_{2}$. We have patterned micronsized structures of chromium, titanium, silicon dioxide, and silicon nitride using this same technique.

\section{B. Spherical target substrates}

The spherical printing method described in Sec. IV B was used to print thin films on flexible substrates deformed into spherical caps. Sturm et al. ${ }^{27}$ have recently shown how to pattern thin-film circuits into small islands on a flat substrate, which is then spherically deformed. Use of these device islands prevents cracking of the brittle semiconductor and insulator films which normally occurs during the inflation procedure described above. In this work, we have focused on patterning metal films for interconnecting such device islands on spherical surfaces by printing and wet etching.

The top micrograph in Fig. 9 depicts an array of PS squares letterpress printed onto the gold-coated concave side of a spherically deformed polyimide substrate. The bottom image depicts the sample surface after wet etching the $90 \mathrm{~nm}$ gold and $10 \mathrm{~nm}$ chromium layers and stripping the polystyrene mask. The cracks in the gold film were formed during the first inflation step when the gold coated foil was spheri- 


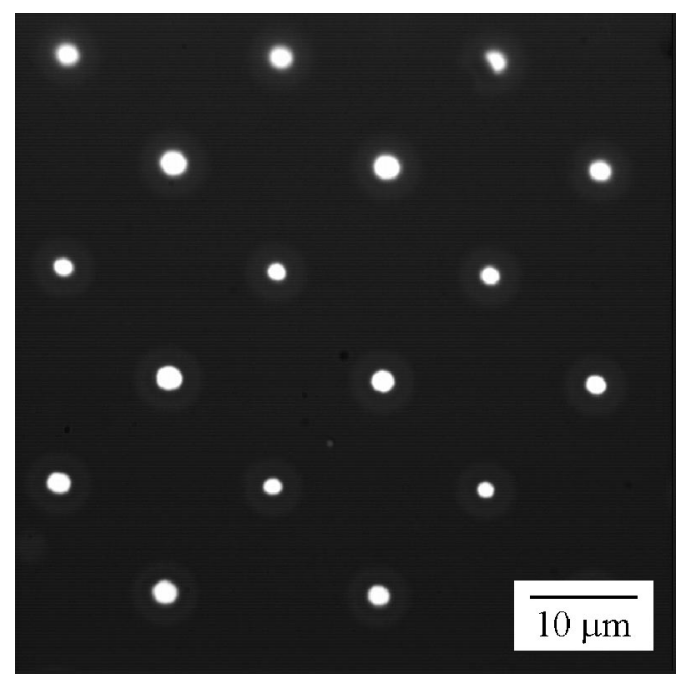

FIG. 8. Optical micrograph showing an array of 2.5 - $\mu \mathrm{m}$-diam gold dots with a pitch of $14 \mu \mathrm{m}$ on a glass slide. The dots were formed by printing a polystyrene mask and then wet etching the metal. The $90 \mathrm{~nm}$ gold layer sits atop a $10 \mathrm{~nm}$ chromium adhesion layer.

cally deformed. Printing the polystyrene patterns in a spherical geometry required a slightly elevated heating temperature $\left(T=200^{\circ}\right)$ in order to make the stamp retract spontaneously from the target substrate after printing. At lower temperatures, the two surfaces separated in a region surrounding the apex but not in the annular region near the clamped edges where the elastic restoring force was likely insufficient to overcome the adhesive force of the polymer.

The quality of the printed structures is similar to those obtained with flat target substrates; however, the spherically printed patterns, especially those with sharp corners, become more rounded. Those with the smallest feature sizes also undergo more spreading. Both of these effects lead to more pronounced degradation in pattern fidelity. The higherprocessing temperature required for spherical printing causes a reduction in polymer viscosity and therefore more rapid film spreading. The patterns also spread for a longer period of time since the inflation apparatus, as currently designed, cools rather slowly. Various improvements are currently being investigated.

\section{SUMMARY}

We have developed a letterpress technique capable of directly printing micrometer sized polymer films onto flat and spherical surfaces. Patterned films shaped as squares or dots are shown to serve as reasonable masks against etching by wet or dry processes. Various thermoplastic polymer films can be printed with this technique so long as the processing temperature exceeds the melting point for crystalline polymers or the glass transition point for amorphous polymers. Image transfer from the printing plate to a target substrate does not rely on a large applied pressure since the polymer film remains in its liquefied state during contact. This printing method allows the formation of structures on surfaces that are chemically or physically heterogeneous; it can also
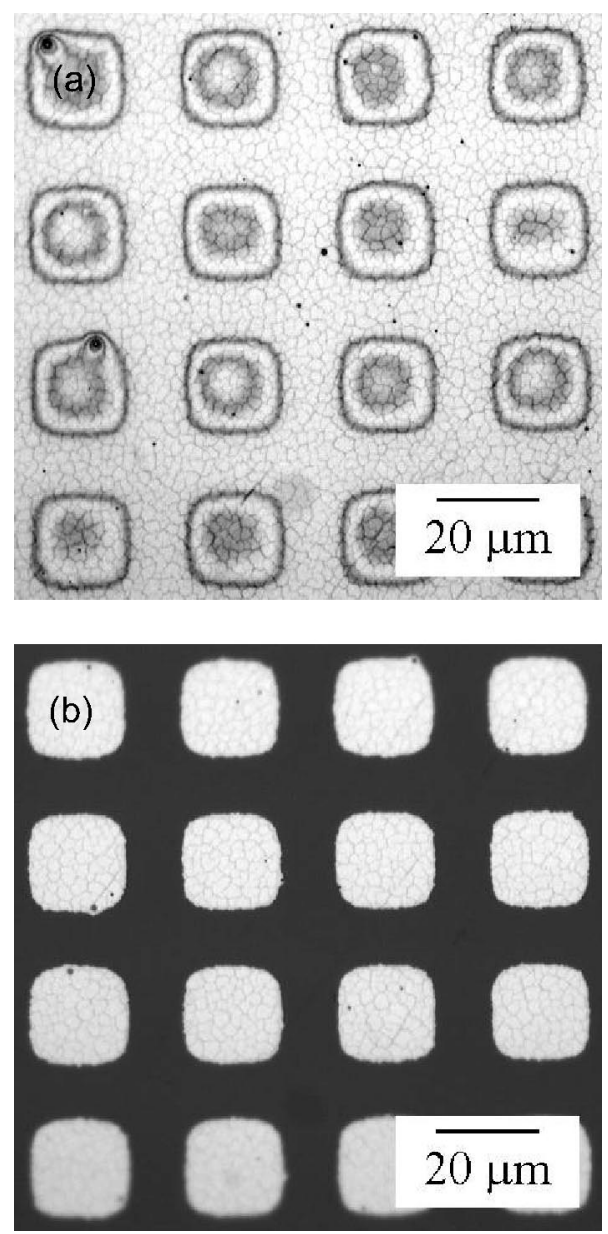

FIG. 9. Optical micrograph of an array of polystyrene squares printed onto the gold coated, concave side of a spherically deformed polyimide substrate. The bottom image shows the same region of the foil after wet etching the gold layer and stripping the polystyrene. The cracks in the gold film appeared when the foil was initially deformed into a sphere and are not a result of the printing step. The $90 \mathrm{~nm}$ gold film sits atop a $10 \mathrm{~nm}$ chromium adhesion layer.

be modified for use in a roller configuration, making it particularly adaptable to flexible, curved, and large area formats. The inflation procedure provides one possible method for printing on spherically deformed substrates, an achievement not possible with photolithographic techniques. Although pattern fidelity and edge resolution still need improvement, the letterpress technique holds promised for applications that do not require fabrication of nanometer sized features.

\section{CHALLENGES}

While the method, apparatus, and materials being used to demonstrate the power and flexibility of letterpress printing will continue to be improved, there remain certain fundamental challenges. For example, when printing sparse patterns whose spacing is greater than $\sim 500 \mu \mathrm{m}$, flexible stamps have a tendency to sag during inking and printing resulting in the transfer of extraneous polymer to undesired regions. Denser patterns seem not to suffer this problem. The effect of thermal expansion on pattern fidelity is expected to 
be a crucial issue when aligning a printed patterned film with a preexisting film on a very large target substrate. The alignment of embossed features on spherical substrates requires further in-depth study since pattern transfer by spherical inflation and subsequent plate detachment introduces angulardependent shear stresses. dependence. Pattern distortion induced by ink heating and spreading must also be minimized. Perhaps the most basic and interesting question pertinent to all printing techniques involves the fundamental limit of resolution attainable with this direct contact method. We have recently made some headway on this front by identifying three separate regimes during the spreading of printed, molten asymmetric patterns. This type of information can be used in "reverse-engineering" a certain printed shape in order to achieve the desired final pattern. While these and more challenges lie ahead, the exploratory studies presented here provide encouraging evidence for the possibility of using contact printing techniques as a cheap, fast, and versatile alternative to current photolithographic printing.

\section{ACKNOWLEDGMENTS}

This project was funded by the Electronic Technology Office of the Defense Advanced Research Projects Agency (DARPA) through the Molecular Level Printing program and by the New Jersey Commission on Science and Technology. We also wish to thank the Eastman Kodak Corporation for their generous support through the Kodak Graduate Fellowship Program (SMM). Iris Hsu and Professor James Sturm of the Department of Electrical Engineering kindly lent us the apparatus for spherical deformation of flexible foils used in this study.

${ }^{1}$ Pocket Pal, 18 th ed., edited by M. H. Bruno (International Paper, Memphis, 2000), pp. 22, 135.

${ }^{2}$ A. Kumar, H. A. Biebuyck, and G. M. Whitesides, Langmuir 10, 1498 (1994).
${ }^{3}$ R. C. Tiberio et al., Appl. Phys. Lett. 62, 476 (1993).

${ }^{4}$ Y. Mikami et al., IEEE Trans. Electron Devices 41, 306 (1994).

${ }^{5}$ M. Lahti, S. Leppävuori, and V. Lantto, Appl. Surf. Sci. 142, 367 (1999).

${ }^{6}$ F. Garnier, R. Hajlaoui, A. Yassar, and P. Srivastava, Science 265, 1684 (1994).

${ }^{7}$ J. A. Rogers, Z. Bao, and V. R. Raju, Appl. Phys. Lett. 72, 2716 (1998).

${ }^{8}$ H. Gleskova, S. Wagner, and D. S. Shen, IEEE Electron Device Lett. 16, 418 (1995).

${ }^{9}$ H. Gleskova and S. Wagner, Mater. Lett. 52, 150 (2002).

${ }^{10}$ T. R. Hebner, C. C. Wu, D. Marcy, M. H. Lu, and J. C. Sturm, Appl. Phys. Lett. 72, 519 (1998).

${ }^{11}$ H. Sirringhaus et al., Science 290, 2123 (2000).

${ }^{12}$ C. M. Hong and S. Wagner, IEEE Electron Device Lett. 21, 384 (2000).

${ }^{13}$ Y. Xia, E. Kim, and G. M. Whitesides, Chem. Mater. 8, 1558 (1996).

${ }^{14}$ M. K. Erhardt, H.-C. Jin, J. R. Abelson, and R. G. Nuzzo, Chem. Mater. 12, 3306 (2000).

${ }^{15}$ R. J. Jackman, J. L. Wilbur, and G. M. Whitesides, Science 269, 5224 (1995).

${ }^{16}$ J. A. Rogers, R. J. Jackman, G. M. Whitesides, J. L. Wagener, and A. M. Vengsarkar, Appl. Phys. Lett. 70, 7 (1997).

${ }^{17}$ Z. Bao, J. A. Rogers, and H. Katz, J. Mater. Chem. 9, 1895 (1999).

${ }^{18}$ A. A. Darhuber, S. M. Troian, and S. Wagner, J. Appl. Phys. 90, 3602 (2001).

${ }^{19}$ H. Gokan, S. Esho, and Y. Ohnishi, J. Electrochem. Soc. 130, 143 (1983).

${ }^{20}$ C. K. Harrison, M. Park, P. M. Chaikan, R. A. Register, and D. H. Adamson, J. Vac. Sci. Technol. B 16, 544 (1998).

${ }^{21}$ T. G. Fox and P. J. Flory, J. Appl. Phys. 21, 581 (1950).

${ }^{22}$ S. Wu, J. Phys. Chem. 74, 632 (1970).

${ }^{23}$ CRC Handbook of Chemistry and Physics, 63 rd ed. edited by R. C. Weast (CRC Press, Boca Raton, FL, 1982), p. C-783.

${ }^{24}$ A. V. Chadov and E. D. Yakhnin, Kolloidn. Zh. 41, 817 (1979).

${ }^{25}$ E. D. Yakhnin and A. V. Chadov, Kolloidn. Zh. 45, 1183 (1983).

${ }^{26}$ P.-h. I. Hsu, M. Huang, S. Wagner, Z. Suo, and J. C. Sturm, Mater. Res. Soc. Symp. Proc. 621, Q8.6 (2001).

${ }^{27}$ J. C. Sturm et al., Mater. Res. Soc. Symp. Proc. 636, D11.4 (2001).

${ }^{28}$ R. J. Fields and M. F. Ashby, Philos. Mag. 33, 33 (1976).

${ }^{29}$ S. M. Miller, A. A. Darhuber, S. M. Troian, and S. Wagner, Mater. Res. Soc. Symp. Proc. 624, 47 (2001).

${ }^{30}$ A. A. Darhuber, S. M. Troian, S. M. Miller, and S. Wagner, J. Appl. Phys. 87, 7768 (2000). 